OPEN ACCESS

Edited by: Enza Palazzo,

University of Campania Luigi

Vanvitelli, Italy

Reviewed by:

Dario Siniscalco,

University of Campania Luigi Vanvitelli, Italy

Rocco Gogliotti,

Vanderbilt University, United States

Nicole Marie Fisher,

Vanderbilt University,

Nashville, United States,

in collaboration with reviewer $R G$

*Correspondence:

Qiang Zhou

zhouqiang@pkusz.edu.cn

${ }^{t}$ These authors have contributed equally to this work

Received: 12 September 2019 Accepted: 13 November 2019

Published: 28 November 2019

Citation: Gao X, Zheng R, Ma X, Gong Z, Xia D and Zhou Q (2019) Elevated Level of $P K M \zeta$ Underlies the Excessive

Anxiety in an Autism Model.

Front. Mol. Neurosci. 12:291. doi: 10.3389/fnmol.2019.00291

\section{Elevated Level of PKM $\zeta$ Underlies the Excessive Anxiety in an Autism Model}

\author{
Xiaoli Gao ${ }^{1 \dagger}$, Rui Zheng ${ }^{1 \dagger}$, Xiaoyan $\mathrm{Ma}^{1}$, Zhiting Gong ${ }^{1,2}$, Dan Xia ${ }^{1,3}$ and Qiang Zhou ${ }^{1,4 *}$ \\ ${ }^{1}$ School of Chemical Biology and Biotechnology, Peking University Shenzhen Graduate School, Shenzhen, China, \\ ${ }^{2}$ Department of Anatomy, College of Preclinical Medicine, Dali University, Dali, China, ${ }^{3}$ Department of Child Healthcare, \\ Shenzhen Children's Hospital, Shenzhen, China, ${ }^{4}$ State Key Laboratory of Chemical Oncogenomics, School of Chemical \\ Biology and Biotechnology, Peking University Shenzhen Graduate School, Shenzhen, China
}

Anxiety affects the life quality of a significant percentage of autism patients. To understand the possible biological basis of this high anxiety level, we used a valproic acid (VPA) model of autism. Anxiety level is significantly higher in VPA-injected mice, at both P35 and P70. In addition, protein kinase $\mathrm{M \zeta}$ (PKM $\zeta$ ) level in the basolateral amygdala $(B\llcorner A)$ is significantly higher in VPA mice at both ages. Consistent with this finding, infusion of a PKM -blocking peptide z-pseudosubstrate inhibitory peptide (ZIP) into BLA significantly reduced anxiety levels in VPA mice. Furthermore, viral overexpression of PKM $\zeta$ in the BLA led to elevated anxiety level in Wild Type (WT) mice, with concomitant higher intrinsic excitability of BLA excitatory neurons. Altogether, our results indicate

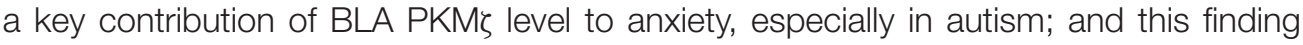
may provide a further understanding of the pathogenesis as well as treatment of anxiety symptoms in autism patients.

Keywords: autism, valproic acid, protein kinase $\mathrm{M} \zeta$, anxiety, basolateral amygdala

\section{INTRODUCTION}

Anxiety is a prominent feature in patients with autistic spectrum disorders (ASDs) since it has been reported that $80 \%$ of ASD patients exhibit significant anxiety level (Sharma et al., 2014; Opoka and Lincoln, 2017). There is considerable evidence that children and adolescents with ASD are at increased risk of anxiety and anxiety disorders (Skokauskas and Gallagher, 2010). It has been estimated that nearly $40 \%$ of ASD children and adolescents may have elevated levels of anxiety or at least one type of anxiety disorders (van Steensel et al., 2011). Hence, better understanding of the biological underpinning of anxiety in ASD is of great importance for both pathogenesis and treatment.

Previous studies have shown that mice exposed to valproic acid (VPA) prenatally around embryonic day (E) 12.5 display abnormalities in their neurological and behavioral development recapitulates many of the core symptoms of ASD, including impaired social behaviors ( $\mathrm{Wu}$ et al., 2017), increased repetitive behaviors (Wang et al., 2018) and increased excitatory to inhibitory ratio (Banerjee et al., 2014), elevated physiological and behavioral measures of anxiety (Edalatmanesh et al., 2013; Kerr et al., 2013; Olexová et al., 2016), and enhanced responsivity to sensory stimulation (Fontes-Dutra et al., 2018). Neural circuits in key brain regions 
implicated in ASD, such as the amygdala, are altered in VPA model animals and thus they are a useful model to investigate circuitry malfunctions in emotional and behavioral abnormalities, especially anxiety.

Abnormal functioning of the amygdala has long been implicated in the etiology of ASD (Kerr et al., 2013), and it is of the major brain structures contributing to anxiety (Bertelsen et al., 2017). Behavioral phenotypes associated with anxiety disorders are accompanied by alterations in GABAergic transmission in the amygdala (Wu et al., 2018), such as low GABA levels (Nemeroff, 2003; Muller et al., 2007). Thus, hyperexcitability of amygdala may directly contribute to the genesis of anxiety. Elevated expression of GluN2A- and GluN2B-NMDA receptors and augmented long-term potentiation (LTP) were found in neocortex of VPA mice indicating increased excitatory synaptic functions (Wang et al., 2018).

To further identify potential mediators of this hyperactivity in the amygdala, we decide to focused on protein kinase $\mathrm{M} \zeta$ $(\mathrm{PKM} \zeta)$, which is an atypical protein kinase $\mathrm{C}(\mathrm{PKC})$ isoform implicated in the protein synthesis-dependent maintenance of LTP and memory storage in the brain (Chen et al., 2014; Xue et al., 2015). The constitutive activity of $\mathrm{PKM} \zeta$ has been shown to maintain LTP as inhibiting its activity diminishes LTP (Naik et al., 2000; He et al., 2011). PKM $\zeta$ is mainly expressed in the pyramidal cells in the hippocampal region and neocortex, granular cells in dentate gyrus, and Purkinje cells in cerebellum (Naik et al., 2000). In hippocampus (HPC), $\mathrm{PKM} \zeta$ is distributed widely in the soma and dendrites, with no expression in the axons of the HPC, and expression is highest in the postsynaptic region and dendrites (Kwapis and Helmstetter, 2014). PKM $\zeta$ is highly expressed in the endoplasmic reticulum, but very low in the Golgi and mitochondria (Howell et al., 2014). Null mice of Prkcz that lose both PKM $\zeta$ and PKC $\zeta$ activity exhibit normal behavior in shuttle box test, basal motor functions and sensory perception, but they display reduced anxiety-like behavior (Lee et al., 2013), suggesting a role of PKM $\zeta$ in anxiety.

In this study, we found that the prominent anxiety level in VPA mice is associated with a high expression of PKM $\zeta$ in the basolateral amygdala (BLA). Functional inhibition of PKM $\zeta$ significantly reduces anxiety level in VPA mice while elevation of PKM $\zeta$ level via viral overexpression in BLA in wild type (WT) mice results in enhanced anxiety and higher intrinsic neuronal excitability. Thus, PKM $\zeta$ level in BLA is highly relevant to the anxiety level, and may contribute to the pathological anxiety in autism patients.

\section{MATERIALS AND METHODS}

\section{Animals}

ICR WT mice were purchased from Guangdong Medical Laboratory Animal Center (Guangdong, China), and all experiments have been approved by the Peking University Shenzhen Graduate School Animal Care and Use Committee and were in accordance with the ARRIVE guidelines on the Care and Use of Experimental Animals. Male and female animals were fed separately and housed in groups of 4-5. All mice were maintained under standard laboratory conditions at $22 \pm 2{ }^{\circ} \mathrm{C}$, with $50 \pm 10 \%$ relative humidity and on a $12 \mathrm{~h}$-light/dark cycle, with food and water made available ad libitum.

Female mice weighing 40-60 g and male mice weighing 40-70 g ICR mice were used. Before any experimental procedure was carried out, animals were acclimated for 1 week in the experimental rooms. Their fertility cycle was controlled, and they were allowed to mate overnight when females were in a pro-estrus state. Vaginal smears on glass slides were examined on the following morning; and if spermatozoa were found, it was designated as first day of pregnancy. Each pregnant mouse was then housed separately and divided into control and VPA-treated groups. VPA (Cat. No. p4543, Sigma-Aldrich, UK) was dissolved in $0.9 \%$ saline at a concentration of $250 \mathrm{mg} / \mathrm{ml}$. Females received a single intraperitoneal injection of $600 \mathrm{mg} / \mathrm{kg}$ sodium valproic (VPA) on E12.5 day after conception, and control females were injected with the same amount of saline at the same time point. Females were housed individually and allowed to raise their own litters. The number of viable offspring born in both groups: control and VPA-treated was normal. All animals exposed to VPA during gestation developed a characteristic "kink" in their tails, which was easily distinguishable from the aged-matched controls. The offspring were weaned on postnatal day (PND) 21. All subsequent experiments were performed only on the male offspring.

\section{Behavioral Testing}

\section{Open Field}

Open field test was conducted on Day 35 (P35) and adult (9-10 weeks, P70). Mice were allowed to acclimate for $1 \mathrm{~h}$ before testing in a quiet room under adjusted lighting. A square wooden box $(100 \mathrm{~cm} \times 100 \mathrm{~cm} \times 40 \mathrm{~cm})$ was used for this locomotor activity test. The floor area was divided into 25 blocks of equal size with nine blocks making up the center grid. Mice were allowed to acclimate in the box for $5 \mathrm{~min}$ and then placed inside the central block and its movements monitored with a video camera for $10 \mathrm{~min}$. The number of blocks that mice passed through (cross grid) and the frequency of straight upward movements (vertical) were recorded. Open field was thoroughly cleaned with 70\% alcohol between test animals.

\section{Elevated Plus Maze (EPM) Test}

The elevated plus maze (EPM) apparatus consisted of two open arms $(30 \times 5 \mathrm{~cm})$, two closed arms of the same size with $15 \mathrm{~cm}$ high walls and a center platform $(5 \times 5 \mathrm{~cm})$. The apparatus was elevated to a height of $35 \mathrm{~cm}$ above the test room floor. Mice were placed in the test room to habituate for 1-2 h. Mice were placed in the central area facing one of the open arms at the start of the test. Time in open arm and number of entries to open arm were recorded for $300 \mathrm{~s}$ using ANY-maze software. The apparatuses were cleaned with $75 \%$ alcohol after each test.

\section{Shuttle (Light/Dark) Box}

Shuttle box consisted of two compartments with different illumination intensity: a light chamber (295 lx) and a dark 
chamber $(0 \mathrm{~lx})$. They were of the same size and shape $(21 \times 21 \times 25 \mathrm{~cm})$ and separated by a Plexiglas wall $(21 \times 25 \mathrm{~cm})$. A hole of $3 \times 5 \mathrm{~cm}$ at the bottom of separating wall connected the two chambers. Mice were allowed to move freely between these two chambers. At the start of the test, mice were placed inside the dark chamber. Locomotion was recorded using a camera placed above the shuttle box, and time spent by each mouse in the light box was measured manually. Shuttle box was cleaned with $30 \%$ isopropanol after each test.

\section{Western Blot Measurements}

Western blotting was used to examine the expression of PKM $\zeta$ in BLA, HPC and medial prefrontal cortex (mPFC). Tissues were collected, homogenized in RIPA buffer containing $1 \mathrm{mM}$ PMSF (Bi Yun Tian, China), $25 \mu \mathrm{M}$ leupeptin (Sigma), and $1 \mu \mathrm{g} / \mathrm{ml}$ aprotinin (Sigma), centrifuged at $4^{\circ} \mathrm{C}$ for $0.5 \mathrm{~h}$ at $13,000 \mathrm{~g}$, with the supernatant collected. Total protein lysates made from different encephalic regions were mixed with SDS gel-loading buffer and heated for $5 \mathrm{~min}$ at $100^{\circ} \mathrm{C}$. Samples $(15 \mu \mathrm{g}$ protein in each group) were separated on 12\% SDS-PAGE gels (Invitrogen, Carlsbad, CA, USA), and transferred to polyvinylidene difluoride membranes (Millipore, Bedford, MA, USA). The membranes were blocked for $1 \mathrm{~h}$ at room temperature with $5 \%$ nonfat milk in TBST (TBS containing 0.05\% Tween 20) and then probed with specific primary antibody (anti-PKM $\zeta, 1: 2,000$, Cat. No. JH6065, Covance, UK, Volk et al., 2013) for overnight at $4^{\circ} \mathrm{C}$. A horseradish peroxidase (HRP)-conjugated secondary antibody was then added for $2 \mathrm{~h}$ at room temperature. Immuno-positive $\mathrm{PKM} \zeta$ bands were scanned and densitometrically analyzed by automated ImageJ software (NIH Image, Version 1.61), and their total protein densities were expressed relative to GAPDH signals.

\section{Peptide Infusion}

To inhibit the activity of PKM $\zeta$ in the BLA, mice were deeply anesthetized with isoflurane on the day of surgery. The stereotaxic coordinates for BLA were AP $-1.4 \mathrm{~mm}$ and $\mathrm{ML} \pm 4.0 \mathrm{~mm}$ and $\mathrm{DV}-5.08 \mathrm{~mm}$. The PKM $\zeta$ inhibitor z-pseudosubstrate inhibitory peptide (ZIP) was dissolved in sterile saline and infused at a concentration of $10 \mathrm{mM}$. ZIP or saline were infused into BLA (400 nl per hemisphere) at a rate of $80 \mathrm{nl} / \mathrm{min}$. The injection needle was left in place for an additional $5 \mathrm{~min}$.

\section{Viral Transfection}

To overexpress PKM $\zeta$ in the BLA, mice were deeply anesthetized with isoflurane and injected with virus. Three-hundred nanoliter of rAAV-hSyn-GFP-pA virus (Brain VTA Technology Company Limited, Wuhan, China) at an injection speed of $80 \mathrm{nl} / \mathrm{min}$ was injected bilaterally in the BLA area (BLA; $-1.4 \mathrm{AP}$, $\pm 4.0 \mathrm{ML},-5.08 \mathrm{DV}$; from Bregma). Five-hundred nanoliter of rAAV-hSyn- PKM $\zeta$-pA virus (Brain VTA Technology Company Limited, Wuhan, China) was injected in the BLA unilaterally using a micro syringe pump. Behavioral experiments were conducted 4 weeks after this procedure.

\section{Immunohistochemistry}

To examine whether there is sufficient overexpressed PKM $\zeta$ in the parvalbumin (PV)-positive neurons, we examined the overlap between the expressed of AAV-hSyn-PKM $\zeta$-GFP and PV staining in brain sections. Mice brains were fixed with $4 \%$ (vol/vol) paraformaldehyde (PFA), dehydrated with gradient sucrose (20\% and 30\%) and embedded in optimal cutting temperature compound (OCT). The embedded tissues were then cut sagittal into $30 \mu \mathrm{m}$-thick sections using a freezing-sliding Microtome (Leica, Germany) as described previously (Zheng et al., 2017). Crysections were permeabilized in phosphate buffer containing $0.5 \%$ Triton X-100 (PBST), and incubated with primary antibodies (anti-PV, 1:500, ab11427, Abcam, UK) in blocking solution and incubated at $4{ }^{\circ} \mathrm{C}$ overnight. Primary antibodies were detected using Alexa-Fluor fluorescent dye conjugated secondary antibodies (anti-rabbit; Alexa Fluor 546, A11035, Invitrogen, UK). Sections were then counterstained with DAPI for $10 \mathrm{~min}$. For analysis and quantification of immunoreacted areas, sections were imaged using confocal microscopy (Olympus, Tokyo, Japan).

\section{Electrophysiological Recordings}

Live brain slices were acutely prepared from mouse brains as previously described (Yao et al., 2018). Briefly, mice were anesthetized with sodium pentobarbital (1\%), with brain quickly removed from the skull and transferred into ice-cold cutting artificial cerebrospinal fluid (ACSF) containing (in $\mathrm{mM})$ : 110 choline chloride, 25 D-glucose, $25 \mathrm{NaHCO}_{3}$, 11.6 sodium ascorbate, $7 \mathrm{MgSO}_{4}, 3.1$ sodium pyruvate, $2.5 \mathrm{KCl}$, $1.25 \mathrm{NaH}_{2} \mathrm{PO}_{4}$, and $0.5 \mathrm{CaCl}_{2}$. The coronal brain slices $(400 \mu \mathrm{m})$ were cut using a DTK-1000 tissue slicer (DTK, Japan) in the cold cutting solution. The brain slices containing BLA recovered at $35.2^{\circ} \mathrm{C}$ for $30 \mathrm{~min}$, with ACSF containing (in $\mathrm{mM}$ ): $127 \mathrm{NaCl}$, $25 \mathrm{NaHCO}_{3}, 25 \mathrm{D}$-glucose, $2.5 \mathrm{KCl}, 2 \mathrm{CaCl}_{2}, 1.25 \mathrm{NaH}_{2} \mathrm{PO}_{4}$, and $1 \mathrm{MgCl}_{2}$. Then, brain slices were incubated at room temperature for $1 \mathrm{~h}$ before recording. The slices were transferred into the recording chamber on an Olympus microscope (BX51WI) equipped with light and fluorescence illumination. The slices were immersed at room temperature with oxygenated ACSF (3 $\mathrm{ml} / \mathrm{min}$ ). Recording pipettes (3-5 M $\Omega$ ) were filled with internal solution containing (in $\mathrm{mM}$ ): 128 potassium gluconate, $10 \mathrm{NaCl}, 10$ HEPES, $4 \mathrm{Na}_{2}$ ATP, $2 \mathrm{MgCl}_{2}$, 0.5 EGTA, 0.4 NaGTP. All solutions used for electrophysiology were equilibrated with 95\% $\mathrm{O}_{2} / 5 \% \mathrm{CO}_{2}$.

To measure excitability, recording in current-clamp mode was used. Neurons were injected with a $500 \mathrm{~ms}$-long current pulse every $4 \mathrm{~s}$. Data were acquired using HEKA EPC10 double patch clamp amplifier (HEKA). Signals were acquired at a sampling rate of $10 \mathrm{kHz}$ and filtered at $2 \mathrm{kHz}$. Neurons with holding current larger than $-200 \mathrm{pA}$ (at $-60 \mathrm{mV}$ ) were excluded from data analysis.

\section{Data Analysis}

Data were analyzed using GraphPad Prism software. Statistical analysis was performed using unpaired $t$-test, paired $t$-test, One-way or Two-way Repeated Measures ANOVA (One-way or Two-way RM ANOVA) followed by Bonferroni post-test which were specifically stated in the "Results" section. All results were shown as Mean \pm SEM. $P<0.05$ was considered statistically significant. 


\section{RESULTS}

\section{High Innate Anxiety in the VPA Mice}

As mentioned in the "Introduction" section, increased anxiety has been reported in VPA models. Thus, we first tested whether anxiety is higher in our VPA mice and if so when this change occurs. Innate anxiety was measured in P35 and P70 VPA mice using light-dark shuttle box and EPM. The reason for not examining mice younger than P35 is that anxiety cannot be reliably measured at those ages under our experimental conditions.

The EPM test showed elevated anxiety in VPA mice of both P35 and P70: (1) shorter time spent in the open arms (Figure 1A, P35, $P<0.05$; Figure 1E; P70, $t_{(28)}=4.68, P<0.01, n=17$ mice); and (2) less times entering into the open arms (Figure 1B, P35, $P<0.01$; Figure 1F, P70, $P<0.05, n=17)$. The above comparisons were made between VPA and vehicle-injected mice.

Measurements in the light-dark shuttle box also indicated elevated anxiety in VPA mice at P35 and P70: (1) less amount of time in the light box (Figure 1C, P35, $P<0.05$; Figure 1G, P70, $P<0.01, n=17)$; and (2) fewer times shuttling between light and dark boxes (Figure 1D, P35, $P<0.01$; Figure 1H, P70, $P<0.05$, $n=17)$. Taken together, these results indicate significant anxiety in the VPA mice of both adolescence and adult, consistent with prior studies and suggest that the above measurements can be used reliably to measure anxiety level in VPA model mice.

\section{Selective Elevated PKM $\zeta$ Level in the BLA, HPC and MPFC of VPA Mice}

$\mathrm{PKM} \zeta$, an isoform of $\mathrm{PKC}$ with persistent activity, is involved in anxiety associated with pain (Zhang et al., 2016; Du et al., 2017). In addition, reducing PKM $\zeta$ activity has been found to be anxiolytic in a PTSD model (Ji et al., 2014). To examine whether PKM $\zeta$ level is significantly altered in VPA mice, we used Western blot from VPA mice of P35 and P70, in three major brain regions that are involved in anxiety, the BLA, HPC and mPFC. A significant elevation in PKM $\zeta$ level was seen in BLA of both P35 and P70 VPA mice, compared to vehicle-injected (Veh) mice (Figures 2A,B; $P<0.05, P<0.01, n=3$ ). In VPA mice, the level of $\mathrm{PKM} \zeta$ was significantly increased in P70 mice compared to P35 mice (Figures 2A,B; $P<0.01, n=3$ ). In HPC, $\mathrm{PKM} \zeta$ level in P70 mice was significantly lower in the VPA mice compared to Veh group (Figures 2C,D; $P<0.05, n=3$ ), but there was no significant difference in $\mathrm{PKM} \zeta$ level between VPA mice and Veh mice at P35 (Figures 2C,D; $P=0.486, n=3$ ). In the mPFC, no significant difference in the $\mathrm{PKM} \zeta$ level was found between the VPA and Veh mice, at both P35 and P70 (Figures 2E,F; $P=0.4911, P=0.9278, n=3$ ). Taken together,
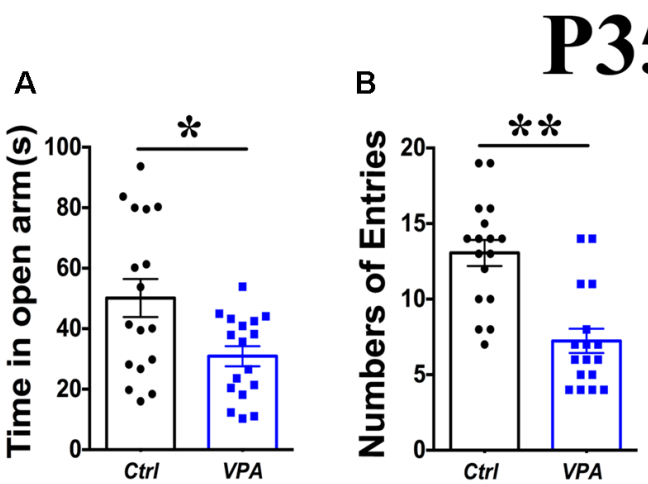

c

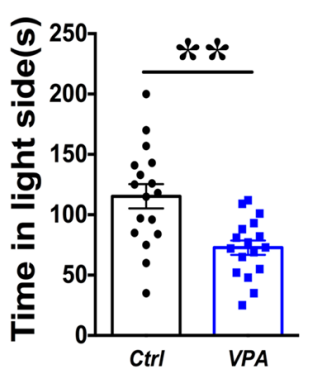

D

P70

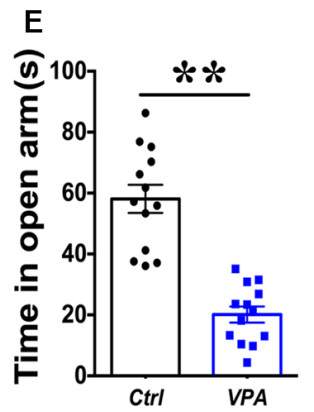

$F$

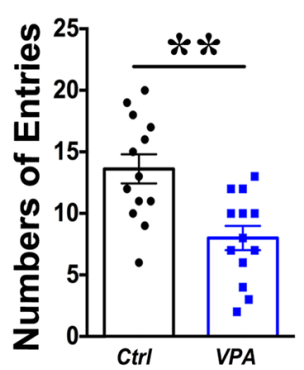

G

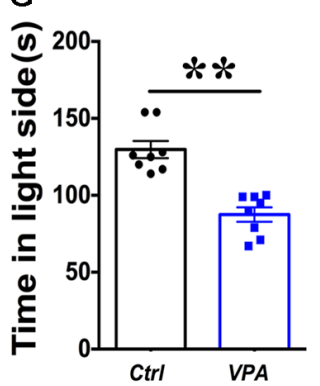

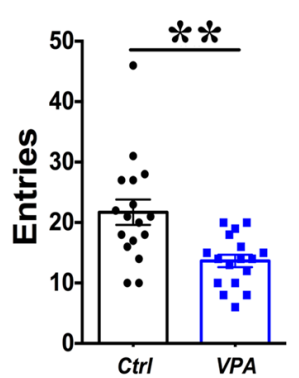

H

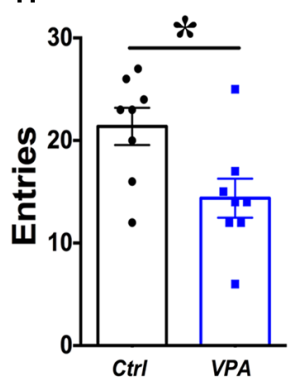

FIGURE 1 | Elevated anxiety in valproic acid (VPA) mice. Time in the open arms (A) and numbers of entries to the open arms (B) on the elevated plus maze (EPM) was significantly reduced in P35 VPA mice compared to the control mice. Time in the light box (C) and entries to the light box (D) was significantly reduced in P35 VPA mice compared to the control mice. Time in the open arms (E) and numbers of entries to the open arms (F) on the EPM was significantly reduced in P70 VPA mice compared to the control mice. Time in the light box (G) and entries to the light box (H) was significantly reduced in P70 VPA mice compared to the control mice. ${ }^{*} P<0.05, * * P<0.01$. 


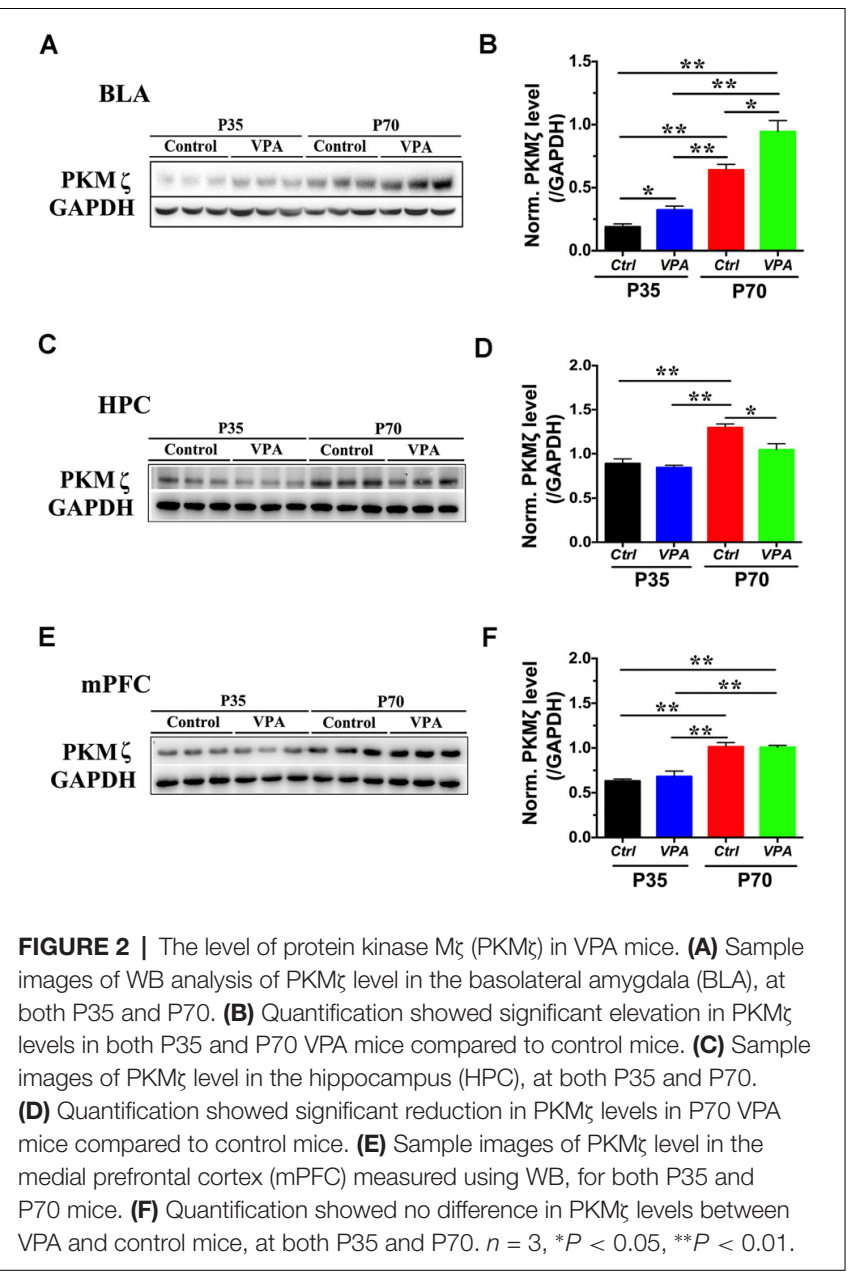

there is a significant and selective elevation in PKM $\zeta$ level in the BLA of VPA mice at both P35 and P70 compared to Veh group.

\section{Reducing PKM $\zeta$ Activity Reduces Anxiety in the VPA Mice}

One likely consequence of higher expression of $\mathrm{PKM} \zeta$ is higher activity of PKM $\zeta$. To further understand whether elevated PKM $\zeta$ has a direct influence on the anxiety level in VPA mice, we inhibited its activity using a well-established method, a short peptide ZIP. Previous studies demonstrated the efficacy of ZIP in reducing the activity of $\mathrm{PKM} \zeta$ and erasure of formed memory (Lin et al., 2013). Bilateral infusion of ZIP into BLA of $30 \mathrm{~min}$ prior to testing showed significantly higher time in the center area (Figure 3A; $P<0.01, n=13$ mice), and there was also a significant increase in locomotion as revealed by longer distance traveled (Figure 3B; $P<0.01, n=13$ ). In the EPM test, there was no significant increase in the open arm time (Figure 3C; $P=0.97, n=13$ ), while numbers of entries to the open arms were significantly increased in the P35 VPA mice compared to those infused with vehicle (Figure 3D; $P<0.05, n=13$ ). In addition, the time in the light side was not altered (Figure 3E; $P=0.16$, $n=13$ ), and entries to the light side (Figure 3F; $P<0.05, n=13$ ) was significantly higher in the ZIP-infused VPA mice, compared to vehicle-injected mice.

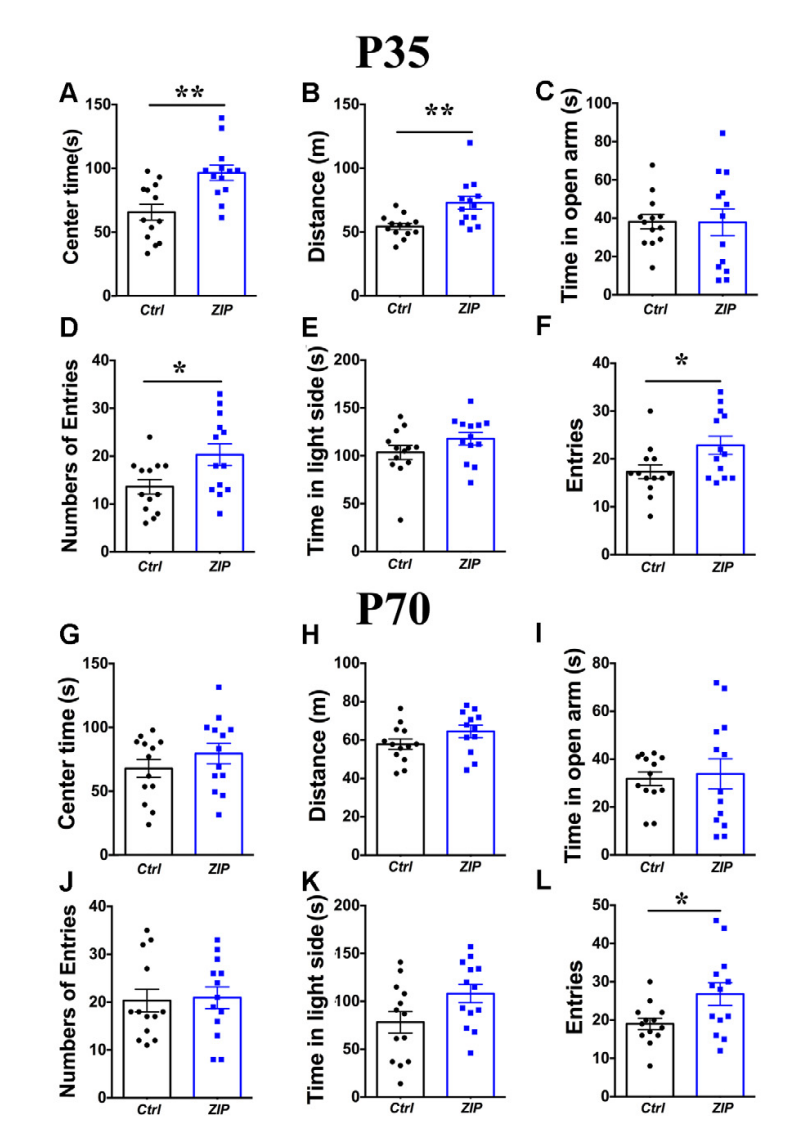

FIGURE 3 | Reducing PKM peptide (ZIP) decreased anxiety in VPA mice. Time in the center (A) in the open field test were significantly increased after infusion of ZIP in the P35 VPA mice compared to those infused with Vehicle. Distance traveled (B) in the open field test were significantly increased after infusion of ZIP in the P35 VPA mice compared to those infused with Vehicle. Time in the open arm (C) was not altered while numbers of entries to the open arms (D) were significantly increased in the P35 VPA mice compared to those infused with vehicle. Time in the light box (E) was not affected while entries to the light box (F) was significantly increased in the P35 VPA mice compared to those infused with vehicle. Time in the center (G) in the open field test were not significantly altered after infusion of ZIP in the P70 VPA mice compared to those infused with vehicle. Distance $\mathbf{( H )}$ traveled in the open field test were not significantly altered after infusion of ZIP in the P70 VPA mice compared to those infused with vehicle. Time in the open arm (I) was not altered and numbers of entries to the open arms $(\mathbf{J})$ were not significantly altered in the P70 VPA mice compared to those infused with Vehicle. Time in the light box (K) was not affected while entries to the light box (L) was significantly increased in the P70 VPA mice compared to those infused with vehicle. ${ }^{*} P<0.05$, ${ }^{* *} P<0.01$

In contrast, the same infusion did not result in significant changes in anxiety in P70 VPA mice. We found no significant impact of ZIP on the center time (Figure 3G; $P=0.28, n=13$ ), and distance traveled was not altered (Figure $3 \mathrm{H} ; P=0.12$; $n=12$ ). We also found that neither time in the open arms (Figure 3I; $P=0.77, n=13$ ) nor numbers of entries to the open arms (Figure 3J; $P=0.85, n=13$ ) in the EPM was altered; nor was time in the light side of shuttle box (Figure 3K; $P=0.06$, $n=13$ ). The only significant change was entries to the light box (Figure 3L; $P<0.05, n=13$ ), Thus, the above results 
suggest that relief of anxiety phenotype in VPA mice appears to be age-dependent.

\section{Overexpression of PKM $\zeta$ in BLA Leads to Higher Anxiety and Elevated Intrinsic Excitability in the WT Mice}

Elevated PKM $\zeta$ level in BLA and amelioration of anxiety in VPA mice by ZIP infusion suggested that BLA PKM $\zeta$ plays a key role in the occurrence of anxiety in VPA model mice. If this is the case, we expect that increasing PKM $\zeta$ in WT mice may result in elevated anxiety. To test this, we overexpressed PKM $\zeta$ using AAV viral transfection in the BLA of P70 WT mice (Figure 4A; Scale bars $=100 \mu \mathrm{m}$ ). Western blot analysis confirmed the effectiveness of this manipulation by showing a significantly higher level of PKM $\zeta$ in mice expressing AAVhSyn-PKM $\zeta$-GFP compared to AAV-hSyn-GFP (Figures 4B,C; $P<0.01, n=5$ mice).

In mice with $\mathrm{PKM} \zeta$ overexpressed, we found significantly reduced center time in the OPT (Figure 4D, $P<0.05$, $n=10$ mice), distance traveled in OPT was not altered (Figure 4E; $P=0.25, n=10$ ), suggesting no alteration in locomotion. We found that time in the open arm not altered (Figure 4F; $P=0.87, n=10$ ) and significantly reduced numbers of entries to the open arms (Figure 4G; $P<0.01, n=10$ ) in the EPM. We also found that significantly reduced time in the light box (Figure 4H; $P<0.01, n=10$ ) and significantly reduced entries to the light box (Figure 4I; $P<0.01, n=10$ ) in the shuttle box test, in mice injected with $\mathrm{PKM} \zeta$ virus compared to those injected with control virus. Put together, these results prove strong evidence for elevated PKM $\zeta$ level in BLA underlies the occurrence of anxiety.

To further elucidate the cellular target of $\mathrm{PKM} \zeta$, we measured intrinsic excitability in the excitatory neurons in BLA of P70 WT mice expressing AAV-hSyn-PKM $\zeta$-GFP or AAV-hSyn-GFP. By injecting a series of depolarizing current steps, we constructed the relationship between current steps and evoked spike frequency. As seen from sample traces (Figure 5A) and population data (Figure 5B), spike frequency is significantly higher in neurons from BLA with $\mathrm{PKM} \zeta$ overexpressed compared to neurons from mice injected with control virus $(P<0.01$, $N=11$ cells $/ 5$ mice). This result indicates that elevated $\mathrm{PKM} \zeta$ may increase the intrinsic excitability of BLA neurons which in turn drives higher anxiety. Since the average spiking frequency in neurons expressing AAV-hSyn-PKM $\zeta$-GFP reached about $40 \mathrm{~Hz}$, we wanted to confirm that they were not the inhibitory PV neurons which are fast-spiking. We stained brain sections with PV (Figure 5C) and found very low overlapping between PV and GFP (Figure 5D), suggesting that they are not PV neurons.

\section{DISCUSSION}

In the present study, we used VPA ASD model mice to examine potential major molecular mechanism underlying the high anxiety phenotype in these mice, in an attempt to understand the genesis of anxiety in ASD. Our findings confirm high

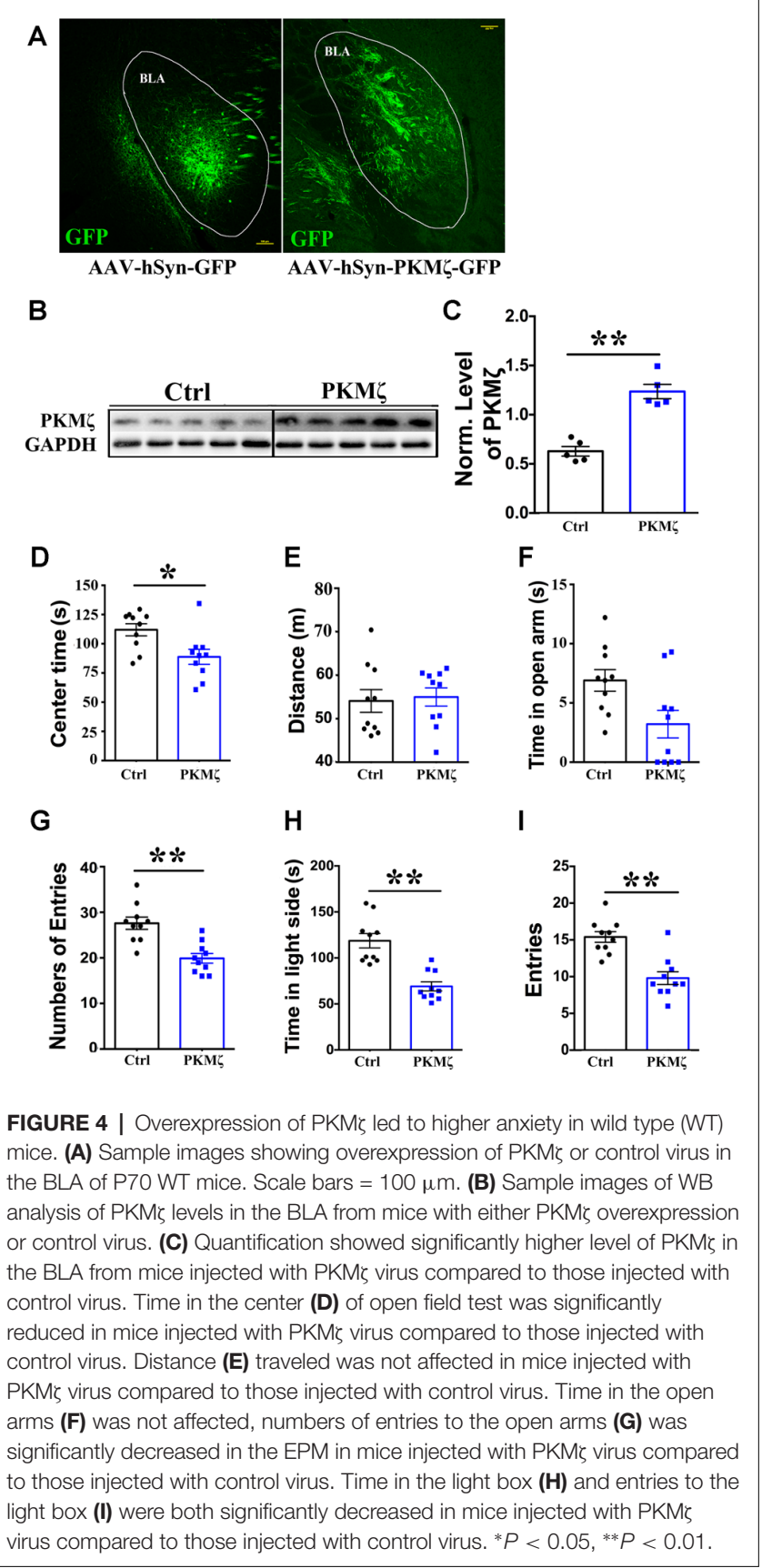

anxiety in VPA mice in both adolescence and adult, and indicate that this anxiety is associated with higher expression of $\mathrm{PKM} \zeta$ in the BLA.

Among the three major brain regions that contribute to anxiety, we found a selective increase in $\mathrm{PKM} \zeta$ level in the $\mathrm{BLA}$, but not in HPC or mPFC. Interestingly, this increase is already significant at P35, the earliest time point, we have tested and still present at P70. This profile of expression suggests that $\mathrm{PKM} \zeta$ level in BLA might be an important contributor to certain phenotypes in the VPA mice. We have selected to focus on anxiety which is quite prominent at both P35 and P70, based on previous studies and our own findings. Supporting a 


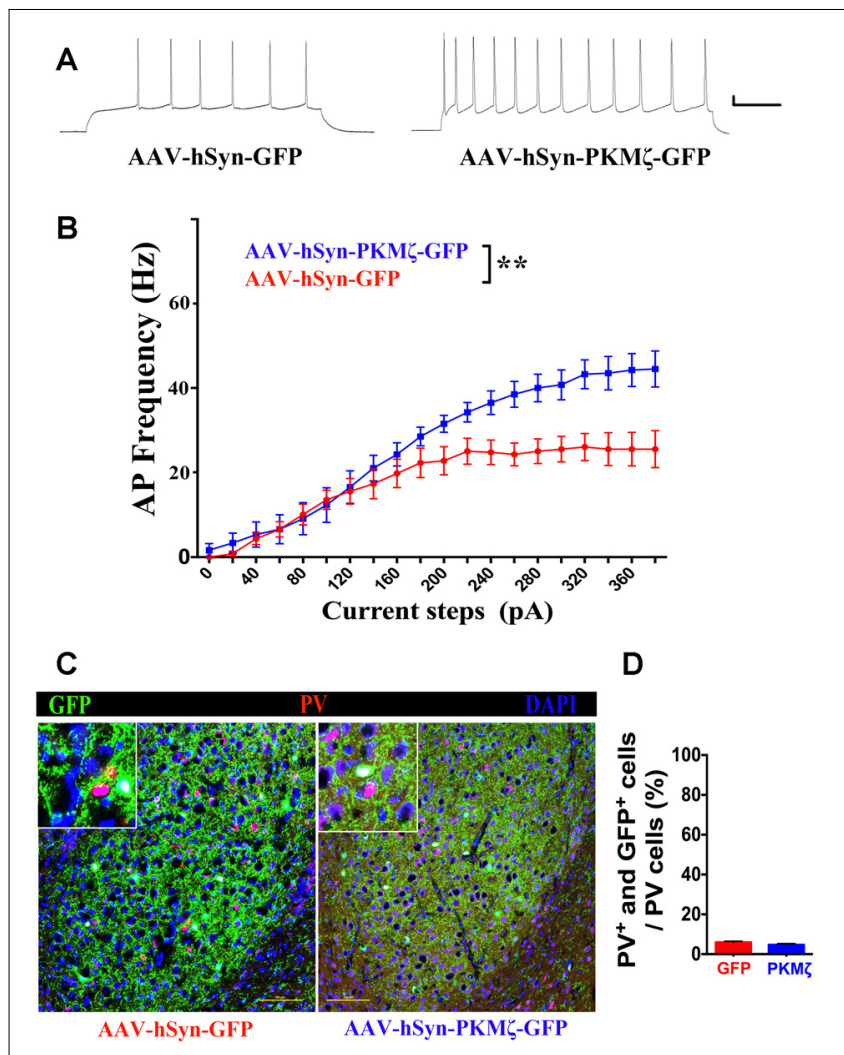

FIGURE 5 | Overexpression of PKM $\zeta$ resulted in higher intrinsic excitability in the excitatory neurons in BLA. (A) Sample spikes evoked by current injection showed higher responses in AAV-hSyn-PKM $\zeta$-GFP (right) than in AAV-hSyn-GFP (left) to the same current step (20 mV, $100 \mathrm{~ms}$ ).

(B) Current-spike frequency relationship showed higher responses in neurons expressing AAV-hSyn-PKM $\zeta$-GFP (blue) than expressing AAV-hSyn-GFP (red). (C) Sample staining of GFP and parvalbumin (PV) in sections from

AAV-hSyn-GFP-expressing (left) and AAV-hSyn-PKM $\zeta$-GFP-expressing brains (right). Scale bars $=100 \mu \mathrm{m}$. (D) Density of neurons that are positive for both GFP and $P V$ is very low. $P=0.1574, N=5$ sections $/$ mice. ${ }^{* *} P<0.01$.

critical role of $\mathrm{PKM} \zeta$ in anxiety in VPA mice, inhibiting $\mathrm{PKM} \zeta$ activity in BLA significantly reduces anxiety levels in P35 VPA mice. In addition, viral overexpression of $\mathrm{PKM} \zeta$ in BLA leads to elevated anxiety level in WT mice, which is associated with higher intrinsic excitability of BLA excitatory neurons. Thus, we have provided substantial evidence on the important contribution of PKM $\zeta$ in BLA to the genesis of anxiety, in both normal mice and ASD model mice. Mounting clinical evidences indicate the prominent presence of anxiety in ASD patients (Ishimoto et al., 2019; Schiltz et al., 2019). We found that reducing PKM $\zeta$ activity with ZIP peptide reduces anxiety in P35 VPA mice but not in P70 VPA mice. Since PKM $\zeta$ level in BLA increases substantially during development and $\mathrm{PKM} \zeta$ level is relatively low at P35 compared to the P70 VPA mice, it is thus possible that locally administered ZIP can effectively inhibit PKM $\zeta$ activity at P35 but not P70. In addition, anxiety in the P70 mice may involve more brain regions than the BLA, while BLA could have a more significant contribution to the genesis of anxiety at P35. It should be noted that there is some debate on whether at the concentration used, ZIP is selective for PKM (Lisman, 2012).
The highly elevated anxiety level in VPA mice allowed us to examine the cellular and molecular basis of this high anxiety. Hyperactivity in the BLA has been implicated in the genesis of anxiety. First, there is a negative correlation between GABAergic markers in the amygdala and anxiety level in that lower GABAergic markers are associated with higher anxiety, implicating an association between BLA hyperactivity and anxiety (Martijena et al., 2002; Flores-Gracia et al., 2010; Quadrato et al., 2014). Second, reducing BLA activity reduces anxiety. Barbalho et al. (2009) showed that infusion of benzodiazepine into BLA significantly reduced anxiety level in both EPM-naïve and EPM-experienced mice, while Sanders and Shekhar (1995) found that inhibiting GABAa receptors in the BLA elevated anxiety level. Third, Olexová et al. (2016) found an elevated mRNA level of type 1 GABA transporter in the BLA of VPA mice together with a high anxiety level. Whether GABAergic transmission is reduced in BLA in VPA mice needs to be tested directly. Banerjee et al. (2013) reported reducing inhibitory transmission in the cortex of VPA mice.

Reduced inhibitory function/transmission likely leads to hyperactivity in BLA, which has been reported in various psychiatric disease and stress models (Sharp, 2017). Another mechanism to cause hyperactivity is higher intrinsic neuronal excitability, as we have examined in this study. Since, we found elevated level of PKM $\zeta$ in BLA, and there is evidence for $\mathrm{PKM} \zeta$ contributing to anxiety (reduced anxiety in the Prkcz null mice (Lee et al., 2013), although the brain regions involved and underlying mechanism not examined), we tested whether there is a link between PKM $\zeta$ level and hyperactivity in the BLA neurons. We showed that overexpression of PKM $\zeta$ leads to higher excitability in BLA excitatory neurons in WT mice, and hence potentially linking $\mathrm{PKM} \zeta$ level in BLA neurons to anxiety. Overexpression of PKM $\zeta$ in the P70 WT mice led to elevated anxiety in them (Figures 4D-I). Thus, these collected evidences provide substantial support for the selective contribution of BLA PKM $\zeta$ to anxiety. Another way that PKM $\zeta$ may lead to hyperactivity is that it may result in stronger synaptic connections to BLA neurons, as Shuette (Chihabi et al., 2016) showed that overexpressing $\mathrm{PKM} \zeta$ increased basal synaptic transmission mediated by increased AMPA receptor trafficking.

What leads to elevated PKM $\zeta$ level in VPA mice? Many studies have shown the importance of $\mathrm{PKM} \zeta$ in the formation and especially maintenance of memory, such as spatial memory and fear memory (Volk et al., 2013). For example, Holliday et al. (2016) showed that reconsolidation of fear memory is associated with elevated level of PKM $\zeta$ in the BLA. PKM $\zeta$ in BLA is also required for the maintenance of contextual fear memory (Kwapis et al., 2009; Volk et al., 2013). Interestingly, Xue et al. (2015) reported that overexpression of PKM $\zeta$ in the prelimbic PFC facilitates the formation of fear memory. Hence, there is a possibility that the more prominent presence of aversive memory (such as fear memory) in VPA mice is caused by elevated PKM $\zeta$ level in BLA of VPA mice, and accumulation of these adverse events may result in high anxiety. In a single prolonged stress model of post-traumatic stress 
disorder, Ji et al. (2014) showed that infusion of ZIP effectively reduced anxiety and depression in these mice, consistent with the notion that PKM $\zeta$ contributes to elevated anxiety caused by stress. Thus, there is a possibility that heightened sensitivity to stress may lead to a higher level of PKM $\zeta$ in VPA mice and anxiety, but this hypothesis needs to be tested directly.

\section{DATA AVAILABILITY STATEMENT}

The datasets generated for this study are available on request to the corresponding author.

\section{ETHICS STATEMENT}

All animal experiments and procedures were approved by the Ethics Committee of Peking University (Permit Number: AP0011).

\section{REFERENCES}

Banerjee, A., Engineer, C. T., Sauls, B. L., Morales, A. A., Kilgard, M. P., and Ploski, J. E. (2014). Abnormal emotional learning in a rat model of autism exposed to valproic acid in utero. Front. Behav. Neurosci. 8:387. doi: 10.3389/fnbeh.2014.00387

Banerjee, A., Garcia-Oscos, F., Roychowdhury, S., Galindo, L. C., Hall, S., Kilgard, M. P., et al. (2013). Impairment of cortical GABAergic synaptic transmission in an environmental rat model of autism. Int. J. Neuropsychopharmacol. 16, 1309-1318. doi: 10.1017/s1461145712001216

Barbalho, C. A., Nunes-de-Souza, R. L., and Canto-de-Souza, A. (2009). Similar anxiolytic-like effects following intra-amygdala infusions of benzodiazepine receptor agonist and antagonist: evidence for the release of an endogenous benzodiazepine inverse agonist in mice exposed to elevated plus-maze test. Brain Res. 1267, 65-76. doi: 10.1016/j.brainres.2009.02.042

Bertelsen, F., Folloni, D., Møller, A., Landau, A. M., Scheel-Krüger, J. R., and Winterdahl, M. (2017). Suppressed play behaviour and decreased oxytocin receptor binding in the amygdala after prenatal exposure to low-dose valproic acid. Behav. Pharmacol. 28, 450-457. doi: 10.1097/FBP.0000000000000316

Chen, Y. W., Lin, H. C., Ng, M. C., Hsiao, Y. H., Wang, C. C., Gean, P. W., et al. (2014). Activation of $\mathrm{mGluR} 2 / 3$ underlies the effects of $\mathrm{N}$-acetylcystein on amygdala-associated autism-like phenotypes in a valproate-induced rat model of autism. Front. Behav. Neurosci. 8:219. doi: 10.3389/fnbeh.2014.00219

Chihabi, K., Morielli, A. D., and Green, J. T. (2016). Intracerebellar infusion of the protein kinase $\mathrm{M}$ zeta (PKM $\zeta$ ) inhibitor zeta-inhibitory peptide (ZIP) disrupts eyeblink classical conditioning. Behav. Neurosci. 130, 563-571. doi: $10.1037 /$ bne0000140

Du, J., Fang, J., Wen, C., Shao, X., Liang, Y., and Fang, J. J. N. P. (2017). The effect of electroacupuncture on PKMzeta in the ACC in regulating anxiety-like behaviors in rats experiencing chronic inflammatory pain. Neural Plast. 2017:3728752. doi: 10.1155/2017/3728752

Edalatmanesh, M. A., Nikfarjam, H., Vafaee, F., and Moghadas, M. (2013). Increased hippocampal cell density and enhanced spatial memory in the valproic acid rat model of autism. Brain Res. 1526, 15-25. doi: 10.1016/j. brainres.2013.06.024

Flores-Gracia, C., Nuche-Bricaire, A., Crespo-Ramírez, M., Miledi, R., Fuxe, K., and Pérez de la Mora, M. (2010). GABA A rho receptor mechanisms in the rat amygdala and its role in the modulation of fear and anxiety. Psychopharmacology 212, 475-484. doi: 10.1007/s00213-010-1973-x

Fontes-Dutra, M., Santos-Terra, J., Deckmann, I., Brum Schwingel, G., DellaFlora Nunes, G., Hirsch, M. M., et al. (2018). Resveratrol prevents cellular and behavioral sensory alterations in the animal model of autism induced by valproic acid. Front. Synaptic Neurosci. 10:9. doi: 10.3389/fnsyn.2018.00009

\section{AUTHOR CONTRIBUTIONS}

QZ, XG and RZ conceived and designed the experiments. RZ analyzed the data and drafted the manuscript. XM, XG, ZG and DX performed the experiments and helped to revise the manuscript. All authors read and approved the final manuscript.

\section{FUNDING}

This work was supported by grants from Shenzhen Science and Technology Innovation Commission Funds (KQTD2015032709315529, JCYJ20170412150845848, JCYJ 20170306165021201) and Sanming Project of Medicine in Shenzhen (SZSM201612036).

\section{ACKNOWLEDGMENTS}

We thank members of the Zhou Lab for helpful discussion.

He, Y. Y., Xue, Y. X., Wang, J. S., Fang, Q., Liu, J. F., Xue, L. F., et al. (2011). PKM $\zeta$ maintains drug reward and aversion memory in the basolateral amygdala and extinction memory in the infralimbic cortex. Neuropsychopharmacology 36, 1972-1981. doi: 10.1038/npp.2011.63

Holliday, E. D., Nucero, P., Kutlu, M. G., Oliver, C., Connelly, K. L., Gould, T. J., et al. (2016). Long-term effects of chronic nicotine on emotional and cognitive behaviors and hippocampus cell morphology in mice: comparisons of adult and adolescent nicotine exposure. Eur. J. Neurosci. 44, 2818-2828. doi: 10.1111/ejn. 13398

Howell, K. K., Monk, B. R., Carmack, S. A., Mrowczynski, O. D., Clark, R. E., and Anagnostaras, S. G. (2014). Inhibition of PKC disrupts addiction-related memory. Front. Behav. Neurosci. 8:70. doi: 10.3389/fnbeh.2014.00070

Ishimoto, Y., Yamane, T., and Matsumoto, Y. (2019). Anxiety levels of children with developmental disorders in japan: based on reports provided by parents J. Autism Dev. Disord. 49, 3898-3905. doi: 10.1007/s10803-019-04092-z

Ji, L. L., Tong, L., Xu, B. K., Fu, C. H., Shu, W., Peng, J. B., et al. (2014). Intra-hippocampal administration of ZIP alleviates depressive and anxiety-like responses in an animal model of posttraumatic stress disorder. Behav. Brain Funct. 10:28. doi: 10.1186/1744-9081-10-28

Kerr, D. M., Downey, L., Conboy, M., Finn, D. P., and Roche, M. (2013). Alterations in the endocannabinoid system in the rat valproic acid model of autism. Behav. Brain Res. 249, 124-132. doi: 10.1016/j.bbr.2013.04.043

Kwapis, J. L., and Helmstetter, F. J. (2014). Does PKM(zeta) maintain memory? Brain Res. Bull. 105, 36-45. doi: 10.1016/j.brainresbull.2013.09.005

Kwapis, J. L., Jarome, T. J., Lonergan, M. E., and Helmstetter, F. J. (2009). Protein kinase Mzeta maintains fear memory in the amygdala but not in the hippocampus. Behav. Neurosci. 123, 844-850. doi: 10.1037/a0016343

Lee, A. M., Kanter, B. R., Wang, D., Lim, J. P., Zou, M. E., Qiu, C., et al. (2013). Prkcz null mice show normal learning and memory. Nature 493, 416-419. doi: 10.1038/nature11803

Lin, H. C., Gean, P. W., Wang, C. C., Chan, Y. H., and Chen, P. S. (2013). The amygdala excitatory/inhibitory balance in a valproate-induced rat autism model. PLoS One 8:e55248. doi: 10.1371/journal.pone.0055248

Lisman, J. (2012). Memory erasure by very high concentrations of ZIP may not be due to PKM-zeta. Hippocampus 22, 648-649. doi: 10.1002/hipo.20980

Martijena, I. D., Rodríguez Manzanares, P. A., Lacerra, C., and Molina, V. A. (2002). Gabaergic modulation of the stress response in frontal cortex and amygdala. Synapse 45, 86-94. doi: 10.1002/syn.10085

Muller, J. F., Mascagni, F., and McDonald, A. J. (2007). Postsynaptic targets of somatostatin-containing interneurons in the rat basolateral amygdala. J. Comp Neurol. 500, 513-529. doi: 10.1002/cne.21185

Naik, M. U., Benedikz, E., Hernandez, I., Libien, J., Hrabe, J., Valsamis, M., et al. (2000). Distribution of protein kinase $M \zeta$ and the complete protein kinase C 
isoform family in rat brain. J. Comp. Neurol. 426, 243-258. doi: 10.1002/10969861(20001016)426:2<243::aid-cne6>3.0.co;2-8

Nemeroff, C. B. (2003). The role of GABA in the pathophysiology and treatment of anxiety disorders. Psychopharmacol. Bull. 37, 133-146.

Olexová, L., Štefánik, P., and Kršková, L. (2016). Increased anxiety-like behaviour and altered GABAergic system in the amygdala and cerebellum of VPA rats-an animal model of autism. Neurosci. Lett. 629, 9-14. doi: 10.1016/j. neulet.2016.06.035

Opoka, S. M., and Lincoln, T. M. (2017). The effect of cognitive behavioral interventions on depression and anxiety symptoms in patients with schizophrenia spectrum disorders: a systematic review. Psychiatr. Clin. North Am. 40, 641-659. doi: 10.1016/j.psc.2017.08.005

Quadrato, G., Elnaggar, M. Y., Duman, C., Sabino, A., Forsberg, K., and Di Giovanni, S. (2014). Modulation of $\mathrm{GABA}_{\mathrm{A}}$ receptor signaling increases neurogenesis and suppresses anxiety through NFATc4. J. Neurosci. 34, 8630-8645. doi: 10.1523/JNEUROSCI.0047-14.2014

Sanders, S. K., and Shekhar, A. (1995). Regulation of anxiety by $\mathrm{GABA}_{\mathrm{A}}$ receptors in the rat amygdala. Pharmacol. Biochem. Behav. 52, 701-706. doi: 10.1016/0091-3057(95)00153-n

Schiltz, H. K., Magnus, B. E., McVey, A. J., Haendel, A. D., Dolan, B. K., Stanley, R. E., et al. (2019). A psychometric analysis of the social anxiety scale for adolescents among youth with autism spectrum disorder: caregiver-adolescent agreement, factor structure, and validity. Assessment doi: 10.1177/1073191119851563 [Epub ahead of print].

Sharma, S., Woolfson, L. M., and Hunter, S. C. (2014). Maladaptive cognitive appraisals in children with high-functioning autism: associations with fear, anxiety and theory of mind. Autism 18, 244-254. doi: $10.1177 / 1362361312472556$

Sharp, B. M. (2017). Basolateral amygdala and stress-induced hyperexcitability affect motivated behaviors and addiction. Transl. Psychiatry 7:e1194. doi: $10.1038 /$ tp.2017.161

Skokauskas, N., and Gallagher, L. (2010). Psychosis, affective disorders and anxiety in autistic spectrum disorder: prevalence and nosological considerations. Psychopathology 43, 8-16. doi: 10.1159/000255958

van Steensel, F. J., Bögels, S. M., and Perrin, S. (2011). Anxiety disorders in children and adolescents with autistic spectrum disorders: a meta-analysis. Clin. Child Fam. Psychol. Rev. 14, 302-317. doi: 10.1007/s10567-011-0097-0

Volk, L. J., Bachman, J. L., Johnson, R., Yu, Y., and Huganir, R. L. (2013). PKM- $\zeta$ is not required for hippocampal synaptic plasticity, learning and memory. Nature 493, 420-423. doi: 10.1038/nature11802
Wang, X., Tao, J., Qiao, Y., Luo, S., Zhao, Z., Gao, Y., et al. (2018). Gastrodin rescues autistic-like phenotypes in valproic acid-induced animal model. Front. Neurol. 9:1052. doi: 10.3389/fneur.2018.01052

Wu, H. F., Chen, P. S., Chen, Y. J., Lee, C. W., Chen, I. T., and Lin, H. C. (2017). Alleviation of $\mathrm{N}$-methyl-D-aspartate receptor-dependent long-term depression via regulation of the glycogen synthase kinase- $3 \beta$ pathway in the amygdala of a valproic acid-induced animal model of autism. Mol. Neurobiol. 54, 5264-5276. doi: 10.1007/s12035-016-0074-1

Wu, H. F., Chen, P. S., Hsu, Y. T., Lee, C. W., Wang, T. F., Chen, Y. J., et al. (2018). D-cycloserine ameliorates autism-like deficits by removing GluA2-containing AMPA receptors in a valproic acid-induced rat model. Mol. Neurobiol. 55, 4811-4824. doi: 10.1007/s12035-017-0685-1

Xue, Y. X., Zhu, Z. Z., Han, H. B., Liu, J. F., Meng, S. Q., Chen, C., et al. (2015). Overexpression of protein kinase $\mathrm{M} \zeta$ in the prelimbic cortex enhances the formation of long-term fear memory. Neuropsychopharmacology 40, 2146-2156. doi: 10.1038/npp.2015.56

Yao, L., Grand, T., Hanson, J., Paoletti, P., and Zhou, Q. (2018). Higher ambient synaptic glutamate at inhibitory versus excitatory neurons differentially impacts NMDA receptor activity. Nat. Commun. 9:4000. doi: 10.1038/s41467018-06512-7

Zhang, Y., Zong, W., Zhang, L., Ma, Y., and Wang, J. J. N. I. (2016). Protein kinase $\mathrm{M} \zeta$ and the maintenance of long-term memory. Neurochem. Int. 99, 215-220. doi: 10.1016/j.neuint.2016.07.007

Zheng, R., Zhang, Z. H., Chen, C., Chen, Y., Jia, S. Z., Liu, Q., et al. (2017). Selenomethionine promoted hippocampal neurogenesis via the PI3K-Akt-GSK3 $\beta$-Wnt pathway in a mouse model of Alzheimer's disease. Biochem. Biophys. Res. Commun. 485, 6-15. doi: 10.1016/j.bbrc.2017. 01.069

Conflict of Interest: The authors declare that the research was conducted in the absence of any commercial or financial relationships that could be construed as a potential conflict of interest.

Copyright (c) 2019 Gao, Zheng, Ma, Gong, Xia and Zhou. This is an open-access article distributed under the terms of the Creative Commons Attribution License (CC BY). The use, distribution or reproduction in other forums is permitted, provided the original author(s) and the copyright owner(s) are credited and that the original publication in this journal is cited, in accordance with accepted academic practice. No use, distribution or reproduction is permitted which does not comply with these terms. 